\title{
Optimal Control of a Threatened Wildebeest-Lion Prey-Predator System in the Serengeti Ecosystem
}

\author{
T. D. Sagamiko', N. Shaban' ${ }^{1}$, C. L. Nahonyo ${ }^{2}$, O. D. Makinde ${ }^{3}$ \\ ${ }^{1}$ Department of Mathematics, University of Dar es Salaam, Dar es Salaam, Tanzania \\ ${ }^{2}$ Department of Zoology and Wildlife Conservation, University of Dar es Salaam, Dar es Salaam, Tanzania \\ ${ }^{3}$ Faculty of Military Science, Stellenbosch University, Stellenbosch, South Africa \\ Email: tsagamiko@gmail.com, shabanmbare@gmail.com, nahonyo@udsm.ac.tz, makinded@gmail.com
}

Received 7 February 2015; accepted 26 March 2015; published 1 April 2015

Copyright (C) 2015 by authors and Scientific Research Publishing Inc.

This work is licensed under the Creative Commons Attribution International License (CC BY).

http://creativecommons.org/licenses/by/4.0/

c) (i) Open Access

\begin{abstract}
We develop a two-species prey-predator model in which prey is wildebeest and predator is lion. The threats to wildebeest are poaching and drought while to lion are retaliatory killing and drought. The system is found in the Serengeti ecosystem. Optimal control theory is applied to investigate optimal strategies for controlling the threats in the system where anti-poaching patrols are used for poaching, construction of strong bomas for retaliatory killing and construction of dams for drought control. The possible impact of using a combination of the three controls either one at a time or two at a time on the threats facing the system is also examined. We observe that the best result is achieved by using all controls at the same time, where a combined approach in tackling threats to yield optimal results is a good approach in the management of wildlife populations.
\end{abstract}

\section{Keywords}

Optimal Control, Prey-Predator System, Threat, Poaching, Serengeti

\section{Introduction}

Population dynamics is the dominant branch of mathematical biology that deals with forces affecting changes in population densities or affecting the form of population growth. It is clear that predator population depends on their prey species for survival and affects the survival and fecundity rate of prey species. Therefore, predator population is affected by changes in prey population in a complex and cyclic predator-prey relationship [1]. 
Many biological species have been driven to extinction and many others are at the verge of extinction due to several external forces such as over exploitation, predation, environmental pollution and mismanagement of the habitat [2]. Thus problems affecting wild animals and their habitats should be evaluated to ensure sustainable conservation of wildlife populations [3].

Environmental pollution, catastrophes and mismanagement of the habitat may cause reduction of species population and probably lead to extinction due to perturbation of the system. Hazards such as fire and drought can also cause the decline of species in an ecosystem [4]. For example, the dry season drought of 1993 in the Serengeti ecosystem is a strong and well studied pertubation. Rainfall during the dry season was only $25 \%$ of the lowest previously recorded level, and directly led to the death of about $30 \%$ of the wildebeest and perhaps $40 \%$ to $50 \%$ of the total park population of the large mammals [5]. However, according to Holling, Serengeti is highly resilient system as it has historically absorbed a wide range of pertubations. Despite being resilient, the time interval for the system to return to its equilibrium seems to be long hence the need of control arises. For example, it took seven years for the wildebeest population to recover from 1993 severe drought and about six years for the lion population from 1994 Canine distemper virus [5].

Kideghesho [6], mentions poaching of wildelife to be among the main threats facing the Serengeti ecosystem. Poaching has become a threat to many migratory populations, particularly as human populations around protected areas increase [7] [8]. It has been reported that local consumption of bushmeat from the Serengeti National Park and surrounding areas is responsible for approximately 70,000 - 129,000 wildebeest deaths per year [9] and any further increase in the amount of poaching could lead to decline in the wildebeest population in the Serengeti-Mara ecosystem [10]. However, lion killing not only in the Serengeti ecosystem but for the entire East Africa is mainly due to Maasai retaliation as lions prey their livestock [11]. In his 19 months study, Kissui [12], recorded 85 lions killings in 12 villages by Maasai retaliation. This happens because many parts of Maasai land have been preserved as wildelife protected areas (e.g. Serengeti, Tarangire and Amboseli) and Game reserves (e.g. Mkomazi and Loliondo) but none of these protected areas are fenced and lions are reported to frequently kill Maasai cattle in adjacent rangelands [13].

Few studies on optimal control of Prey-Predator system such as those by Chakraborty et al., [1], Kar and Ghosh [14] have determined various optimal control strategies. In particular, Kar and Ghosh [14] controlled alternative food to predator to an exploited prey-predator system. However, none of these studies have considered the aspect of drought, poaching and retaliatory killing as threats to be controlled for survival of prey-predator system particularly wildebeest and lions in the Serengeti ecosystem. Therefore, this study intends to apply optimal control theory to maximize Wildebeest-Lion prey-predator population in the Serengeti Ecosytem under threat of drought, poaching and retaliatory killings and ensuring the cost of applying these controls is minimum.

This paper is organized as follows: In Section 2, a threatened prey-predator model is developed. In Section 3, time-dependent controls are introduced and analysis of optimal control is carried out. In Section 4, scenarios for different control strategies are considered and the results are discussed. Lastly, Section 5 presents conclusions about the proposed control strategies suggested for this threatened system. The outcome tends to display a significant increase in the number of individuals due to control strategies employed.

\section{The Model with Threats}

Consider two populations of different species: $x$ a prey population, and $y$, a predator population. The prey species is wildebeest and predator species is lion. Drought affect both species, while wildebeest is threatened by poaching lion is threatened by retaliatory killing. Prey species are assumed to grow logistically to the carrying capacity in the absence of the predator, poaching and drought, hence these are only causes of prey mortality. The rate of increase of the predator population depends on the amount of prey biomass it converts as food. Thus according to Hollying type II functional response [15] the two populations are modeled as follows:

$$
\begin{aligned}
& \frac{\mathrm{d} x}{\mathrm{~d} t}=r x\left(1-\frac{x}{k}\right)-\frac{w x y}{x+a}-p x-(1-f) x, \\
& \frac{\mathrm{d} y}{\mathrm{~d} t}=-a_{2} y+\frac{w_{1} x y}{x+a}-c y-(1-e) y,
\end{aligned}
$$

where $x$ is the prey density at time $t, y$ is the predator density at time $t, r$ is the intrinsic prey growth rate, $k$ is the prey carrying capacity, $w$ is the maximum per capita predation rate, $w_{1}$ is the predator biomass to the prey 
(conversion rate), $p$ is the wildebeest poaching rate in Serengeti, $c$ is lion death rate due to retaliatory killing, $f$ is the percentage of wildebeest resilient to drought, hence $(1-f)$ is the wildebeest death rate due to drought, $e$ is the percentage of lion resilient to drought, hence $(1-e)$ is the lion death rate due to drought, $a$ is the predator half saturation and $a_{2}$ is the lion mortality rate.

\section{The Model with Time Dependent Control Effort}

We introduce into model (1), time dependent control efforts on anti-poaching patrols $\left(u_{1}(t)\right)$, construction of strong bomas $\left(u_{2}(t)\right)$, and construction of dams $\left(u_{3}(t)\right)$ as controls to curtail the threats to the prey-predator system. The prey-predator model (1) thus becomes:

$$
\begin{aligned}
& \frac{\mathrm{d} x}{\mathrm{~d} t}=r x\left(1-\frac{x}{k}\right)-\frac{w x y}{x+a}-\left(1-u_{1}(t)\right) p x-\left(1-u_{3}(t)\right)(1-f) x, \\
& \frac{\mathrm{d} y}{\mathrm{~d} t}=-a_{2} y+\frac{w_{1} x y}{x+a}-\left(1-u_{2}(t)\right) c y-\left(1-u_{3}(t)\right)(1-e) y .
\end{aligned}
$$

\subsection{Analysis of Optimal Control}

The objective is to maximize the species population size at the final time of control while minimizing the cost. These costs are due to control strategies applied such as cost of antipoaching, construction of strong bomas and conservation cost for drought control. We assume the total population $(x+y)$ is to be maximized to the final time, with different relative weights applied to prey and predator populations. For simplicity we take $u_{1}(t)$ as $u_{1}, u_{2}(t)$ as $u_{2}$ and $u_{3}(t)$ as $u_{3}$. Thus the objective function is:

$$
J=\max \left[\left(B_{1} x(T)+B_{2} y(T)\right)-\int_{0}^{T}\left(A_{1} \frac{u_{1}^{2}}{2}+A_{2} \frac{u_{2}^{2}}{2}+A_{3} \frac{u_{3}^{2}}{2}\right) \mathrm{d} t\right],
$$

where $A_{1}, A_{2}, A_{3}, B_{1}, B_{2}$ are positive weights. The term $A_{1} \frac{u_{1}^{2}}{2}$ is the cost of control efforts on antipoaching strategy, $A_{2} \frac{u_{2}^{2}}{2}$ is the cost of control efforts on construction of strong boma and $A_{3} \frac{u_{3}^{2}}{2}$ is the cost of drought control strategy. We seek an optimal control triple $u_{1}^{*}, u_{2}^{*}$ and $u_{3}^{*}$ such that

$$
J\left(u_{1}^{*}, u_{2}^{*}, u_{3}^{*}\right)=\max \left\{J\left(u_{1}, u_{2}, u_{3}\right) \mid u_{1}, u_{2}, u_{3} \in U\right\},
$$

where $U=\left(u_{1}, u_{2}, u_{3}\right)$ such that $u_{1}, u_{2}, u_{3}$ are lebesgue measurable with $0 \leq u_{1} \leq 1,0 \leq u_{2} \leq 1$ and $0 \leq u_{3} \leq 1$ for $t \in[0, T]$ is the control set.

\subsection{Characterization of the Optimal Control}

Pontryagin's maximum principle which provides necessary condition, converts Equations (2) and (3) into a problem of maximizing point-wise a Hamiltonian $H$, with respect to $u_{1}, u_{2}$ and $u_{3}$.

$$
\begin{aligned}
H= & -\left(A_{1} \frac{u_{1}^{2}}{2}+A_{2} \frac{u_{2}^{2}}{2}+A_{3} \frac{u_{3}^{2}}{2}\right)+L_{1}\left\{r x\left(1-\frac{x}{k}\right)-\frac{w x y}{x+a}-\left(1-u_{1}\right) p x-\left(1-u_{3}\right)(1-f) x\right\} \\
& +L_{2}\left\{-a_{2} y+\frac{w_{1} x y}{x+a}-\left(1-u_{2}\right) c y-\left(1-u_{3}\right)(1-e) y\right\},
\end{aligned}
$$

where $L_{1}$ and $L_{2}$ are the adjoint variables or co-state variables.

Theorem 1. For the optimal control tripple $u_{1}^{*}, u_{2}^{*}$ and $u_{3}^{*}$ that maximizes $J\left(u_{1}, u_{2}, u_{3}\right)$ over $U$, then there exists adjoint variables $L_{1}$ and $L_{2}$ satisfying

$$
\frac{\mathrm{d} L_{1}}{\mathrm{~d} t}=-\frac{\partial H}{\partial x}=-\left\{L_{1}\left[r\left(1-\frac{2 x}{k}\right)-\frac{w a y}{(x+a)^{2}}\right]-\left[\left(1-u_{1}\right) p+\left(1-u_{3}\right)(1-f)\right]+L_{2}\left(\frac{w_{1} a y}{(x+a)^{2}}\right)\right\}
$$




$$
\frac{\mathrm{d} L_{2}}{\mathrm{~d} t}=-\frac{\partial H}{\partial y}=-\left\{L_{1}\left[\frac{-w x}{(x+a)}\right]+L_{2}\left[-a_{2}+\frac{w_{1} x}{x+a}-\left[\left(1-u_{2}\right) c+\left(1-u_{3}\right)(1-e)\right]\right]\right\}
$$

and with transversality condition as $L_{1}(T)=B_{1}$ and $L_{2}(T)=B_{2}$, see [10].

By optimality condition, we have $\frac{\partial H}{\partial u}=0$ at $u^{*}$,

$$
\text { That is } \frac{\partial H}{\partial u_{1}}=0 \text { at } u_{1}^{*}, \frac{\partial H}{\partial u_{2}}=0 \text { at } u_{2}^{*} \text { and } \frac{\partial H}{\partial u_{3}}=0 \text { at } u_{3}^{*}
$$

but:

$$
\begin{gathered}
\frac{\partial H}{\partial u_{1}}=-A_{1} u_{1}+L_{1} p x=0, \text { at } u_{1}^{*} \\
\text { Hence } u_{1}^{*}=\frac{L_{1} p x}{A_{1}} . \\
\frac{\partial H}{\partial u_{2}}=-A_{2} u_{2}+L_{2} c y=0, \text { at } u_{2}^{*}, \\
\text { Hence } u_{2}^{*}=\frac{L_{2} c y}{A_{2}} . \\
\frac{\partial H}{\partial u_{2}}=-A_{3} u_{3}+L_{1}(1-f) x+L_{2}(1-e) y=0, \text { at } u_{3}^{*}, \\
\text { Hence } u_{3}^{*}=\frac{L_{1}(1-f) x+L_{2}(1-e) y}{A_{3}} .
\end{gathered}
$$

Equivalently, we can represent the optimal control as

$$
\begin{gathered}
u_{1}^{*}=\min \left\{1, \max \left(0, \frac{L_{1} p x}{A_{1}}\right)\right\}, \\
u_{2}^{*}=\min \left\{1, \max \left(0, \frac{L_{2} c y}{A_{2}}\right)\right\}, \\
\text { and } u_{3}^{*}=\min \left\{1, \max \left(0, \frac{L_{1}(1-f) x+L_{2}(1-e) y}{A_{3}}\right)\right\} .
\end{gathered}
$$

The optimal control can be numerically calculated under various parameter sets using a forward-backward sweep method involving fourth order Runge-Kutta procedure. The successive steps are as follows (see example, Kar \& Ghosh, 2012 [14]).

1) Divide the total time interval into $N$ equal subintervals and set the state at different times as $\boldsymbol{x}=\left(x_{1}, x_{2}, \cdots, x_{N+1}\right)$ and the costate variables as $\boldsymbol{L}=\left(L_{1}, L_{2}, \cdots, L_{N+1}\right)$.

2) Assume control takes zero over the time intervals i.e. $\boldsymbol{u}=(0,0, \cdots, 0)$ for starting iteration.

3) Using the initial condition $x(0)=x_{0}$ solve the state according to the ODE with the values of $\boldsymbol{u}$ forwardly.

4) Using the transversality condition $L_{N+1}=L(T)$, (T=final time) and the values for $\boldsymbol{u}$ as well as previously evaluated values for $\boldsymbol{x}$, solve $\boldsymbol{L}$ in time from costate differential equation in backward process.

5) Update the control entering the new $\boldsymbol{x}$ and $\boldsymbol{L}$ through the rule

$$
u=\min \left(u_{\max }, \max \left(u_{\text {sig }}, u_{\text {min }}\right)\right)
$$

where 


$$
u^{*}= \begin{cases}u_{\min } & \text { if } \frac{\partial H}{\partial u}<0 \\ u_{\text {sig }} & \text { if } \frac{\partial H}{\partial u}=0 \\ u_{\max } & \text { if } \frac{\partial H}{\partial u}>0\end{cases}
$$

\section{Numerical Results and Discussion}

In this section we study numerically an optimal control of a threatened prey-predator system.

Let us take the system parameters $e=0.92$ [5], $f=0.85$ [5], $c=0.08$ [11], $w=0.674$ [16], $w_{1}=0.25$ [17], $a_{2}=0.01$ [17], $p=0.01$ [18], $r=1$ [18], $a=1$ [assumed], $k=300$ [calculated], together with the initial guess states $x(0)=40$ and $y(0)=20$. Assume the weights for prey and predator at final time are being kept fixed as $B_{1}=100$ and $B_{2}=150$. However the weights of controls $u_{1}, u_{2}$ and $u_{3}$ are respectively assumed to be $A_{1}=60, A_{2}=10$ and $A_{3}=90$. The weights of state variables are usually assigned depending on their relative importance while those of controls are assigned relative to their cost implications.

Next, we investigate the effect of the following optimal control strategies on the threatened prey-predator population.

- Strategy A: Application of anti-poaching patrols for controlling poaching.

- Strategy B: Construction of strong bomas for control of retaliatory killings.

- Strategy C: Construction of dams for drought control.

- Strategy D: Combination of application of anti-poaching patrols and construction of strong bomas.

- Strategy E: Combination of application of anti-poaching patrols and construction of dams.

- Strategy F: Combination of construction of strong bomas and dams and.

- Strategy G: Combination of application of anti-poaching patrols, construction of strong bomas and dams.

\subsection{Strategy A: Application of Anti-Poaching Patrols for Control of Poaching}

The application of anti-poaching patrols $u_{1}$ is used to optimize the objective function $J$ while we set the con- struction of strong boma $u_{2}$ and construction of dams $u_{3}$ to zero. In Figure 1, the results show a significant difference in the prey population with optimal strategy compared to prey population without control while no effect to predator population as the control is taken only to prey species. From Table 1 we see that if no control measures are taken the wildebeest population would drop to approximately 152 individuals from 300 individuals expected to be attained during these 5 years if there was no threat. With this control strategy the wildebeest population rises to approximately 188, which is a saving of 36 individuals. Lion population goes to about 29 individuals and with optimal anti-poaching patrol the population almost remains the same because the control measure is taken only to prey population as described in the model.

\subsection{Strategy B: Construction of Strong Bomas for Control of Retaliatory Killings}

The construction of strong boma $u_{2}$ is used to optimize the objective function $J$ while we set the application of anti-poaching patrols $u_{1}$ and construction of dams $u_{3}$ to zero. In Figure 2, the results show a significant difference in the predator population with optimal strategy compared to predator population without control while a little decrease in prey species. From Table 1 we see that due to application of construction of strong bomas lion species increases from 29 to 36 individuals which is a saving of 7 lion individuals. Wildebeest population decreases from 152 to 147 individuals and this is caused by predation as a result of increasing number of lions without taking control of all threats to wildebeest.

\subsection{Strategy C: Construction of Dams for Drought Control}

The construction of dams $u_{3}$ is used to optimize the objective function $J$ while we set the application of antipoaching patrols $u_{1}$ and construction of strong boma $u_{2}$ to zero. In Figure 3, the results show a significant difference in the prey and predator populations with optimal strategy compared to prey and predator populations without control. From Table 1 we see that if no control measures are undertaken the wildebeest population 
Table 1. The different final states and total costs for different controls strategies from numerical codes.

\begin{tabular}{cccc}
\hline Strategy & $x(5)$ & $Y(5)$ & Cost \\
\hline No control & 152 & 29 & $1.9603 \mathrm{e}+004$ \\
Strategy A & 188 & 29 & $1.7083 \mathrm{e}+004$ \\
Strategy B & 147 & 36 & $1.9474 \mathrm{e}+004$ \\
Strategy C & 204 & 44 & $1.7764 \mathrm{e}+004$ \\
Strategy D & 180 & 38 & $1.6848 \mathrm{e}+004$ \\
Strategy E & 240 & 44 & $1.5367 \mathrm{e}+004$ \\
Strategy F & 190 & 60 & $1.7998 \mathrm{e}+004$ \\
Strategy G & 223 & 65 & $1.7083 \mathrm{e}+004$ \\
\hline
\end{tabular}
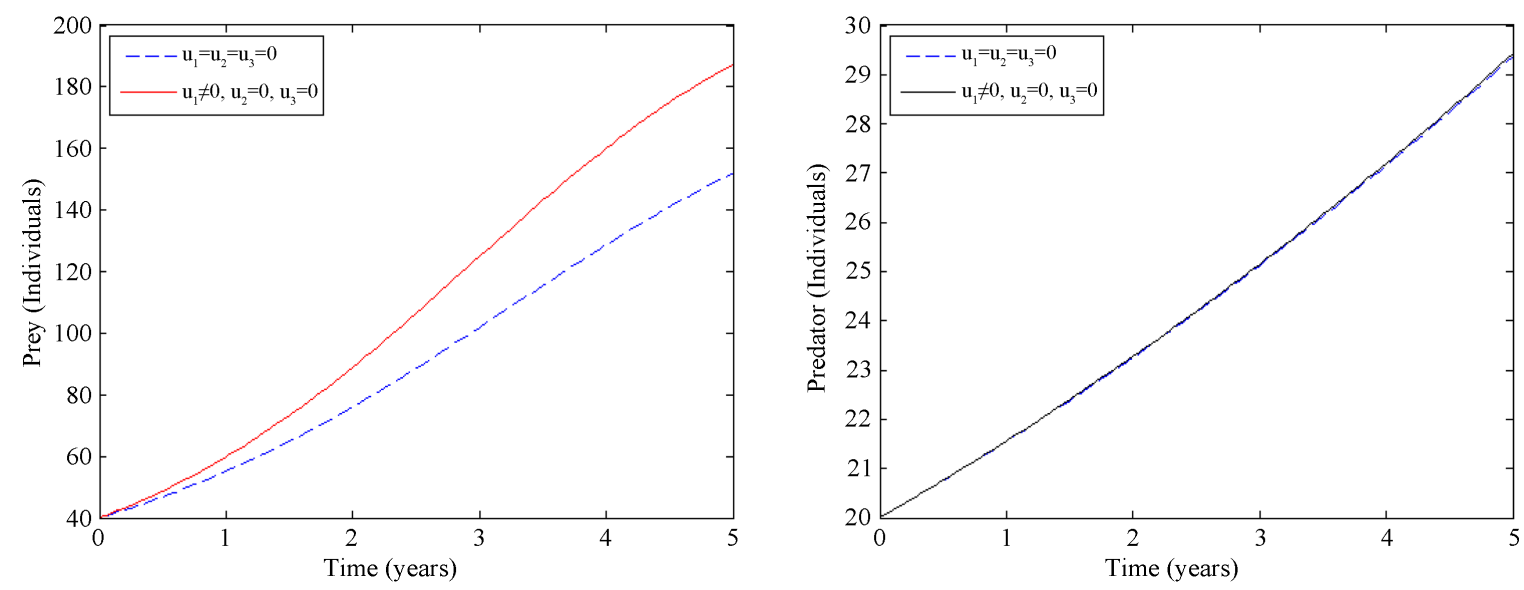

Figure 1. Simulations of a threatened prey-predator model showing the effect of optimal application of anti-poaching patrols.
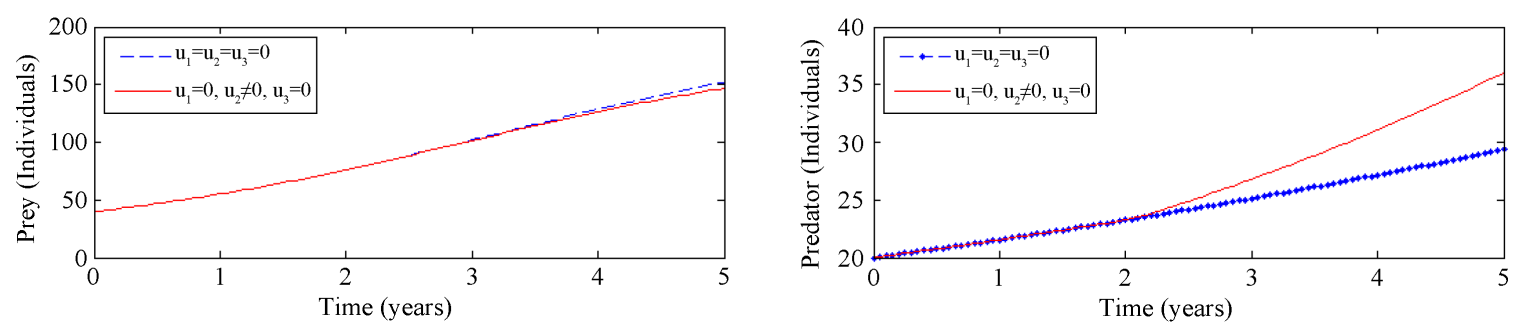

Figure 2. Simulations of a threatened prey-predator model showing the effect of optimal application of construction of strong bomas.

would drop to approximately 152 individuals different from the 300 expected number during the 5 years. With this control strategy the wildebeest population rises to approximately 204, which is a saving of 52 individuals. With optimal construction of dams $\left(u_{3}\right)$ Lion population rises from 29 to 44 individuals at final time of control which is a saving of 15 individuals.

\subsection{Strategy D: Combination of Application of Anti-Poaching Patrols and Construction of Strong Bomas}

The application of anti-poaching patrols $u_{1}$ and construction of strong bomas $u_{2}$ are used to optimize the objective function $J$ while we set construction of dams $u_{3}$ to zero. In Figure 4, the results show a significant difference in prey and predator populations before and after control. From Table 1 numerical results suggests 

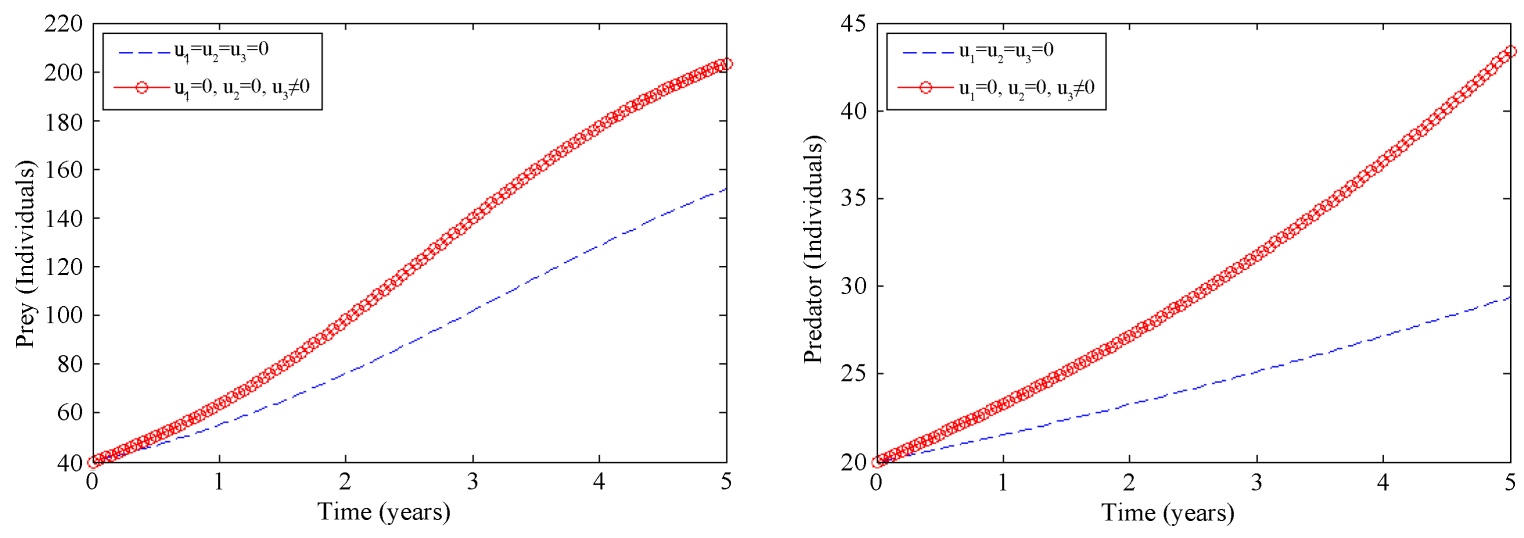

Figure 3. Simulations of a threatened prey-predator model showing the effect of optimal application of construction of dams.
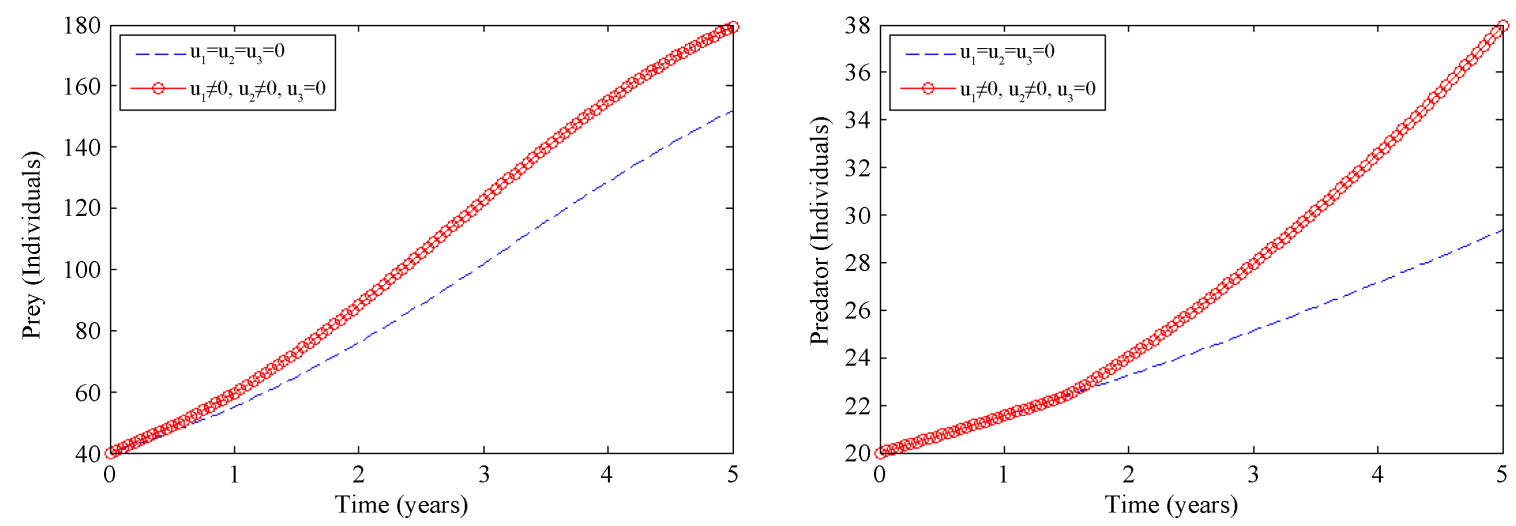

Figure 4. Simulations of a threatened prey-predator model showing the effect of optimal application of anti-poaching patrol and construction of strong bomas.

that if no control measures are applied the lion population would go to 29 individuals, but with this control strategy the population rises to 38 individuals during the 5 years which is a saving of 9 individuals. Wildebeest population rises from approximately 152 to 180 which is a saving of 28 individuals.

\subsection{Strategy E: Combination of Application of Anti-Poaching Patrols and Construction of Dams}

The application of anti-poaching patrols $u_{1}$ and construction of dams $u_{3}$ are used to optimize the objective function $J$ while we set construction of dams $u_{3}$ to zero. In Figure 5, the results show a significant difference in prey and predator populations before and after control. From Table 1 numerical results indicates that if no control measures are taken the lion population would go to 29 individuals during these 5 years. With this control strategy the lion population rises to 44 which is a saving of 15 individuals. Wildebeest population rises from approximately 152 to 240 which is a saving of 88 individuals.

\subsection{Strategy F: Combination of Construction of Strong Bomas and Dams}

The application of construction of strong bomas $u_{2}$ and construction of dams $u_{3}$ are used to optimize the objective function $J$ while we set application of anti-poaching patrol $\mathrm{u}_{1}$ to zero. In Figure 6, the results show a significant difference in prey and predator populations before and after control. From Table 1 numerical results indicate that if no control measures are put in place the lion population would go to approximately 29 individuals from 20 initial individuals during the 5 years. With this control strategy the lion population rises to 60 which is a saving of 31 individuals. Wildebeest population rises from approximately 152 to 190 which is a saving of 38 individuals. 

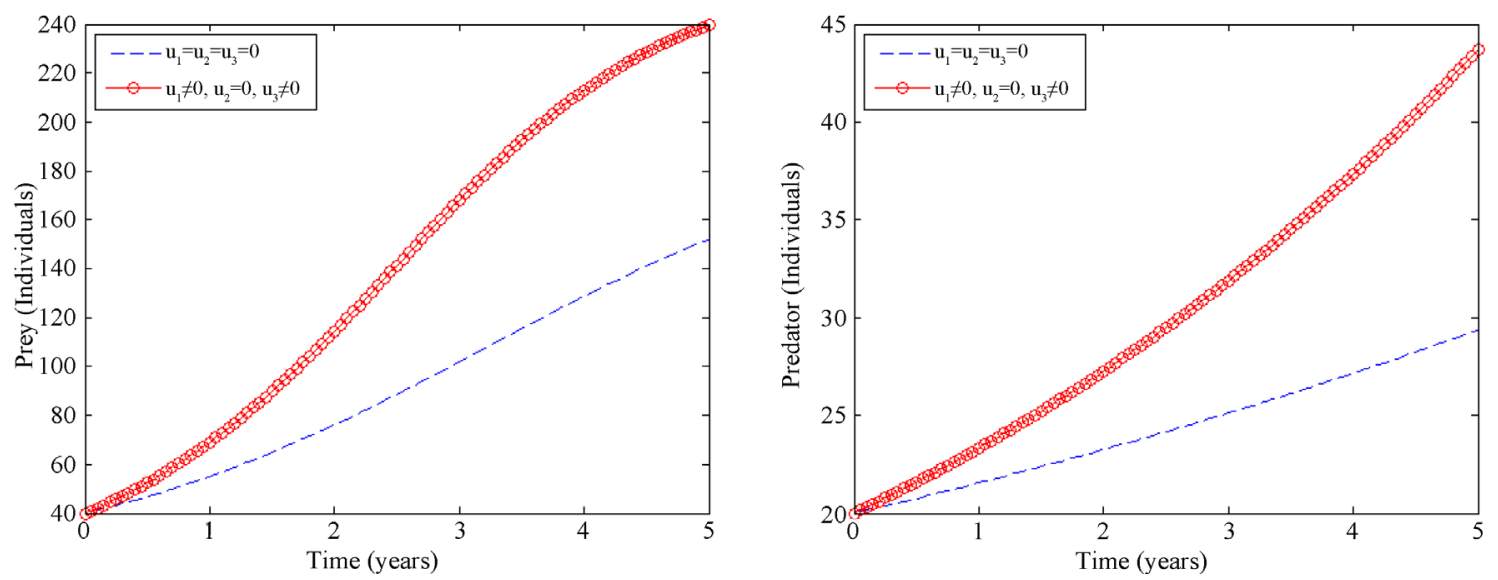

Figure 5. Simulations of a threatened prey-predator model showing the effect of optimal application of anti-poaching patrol and construction of dams.
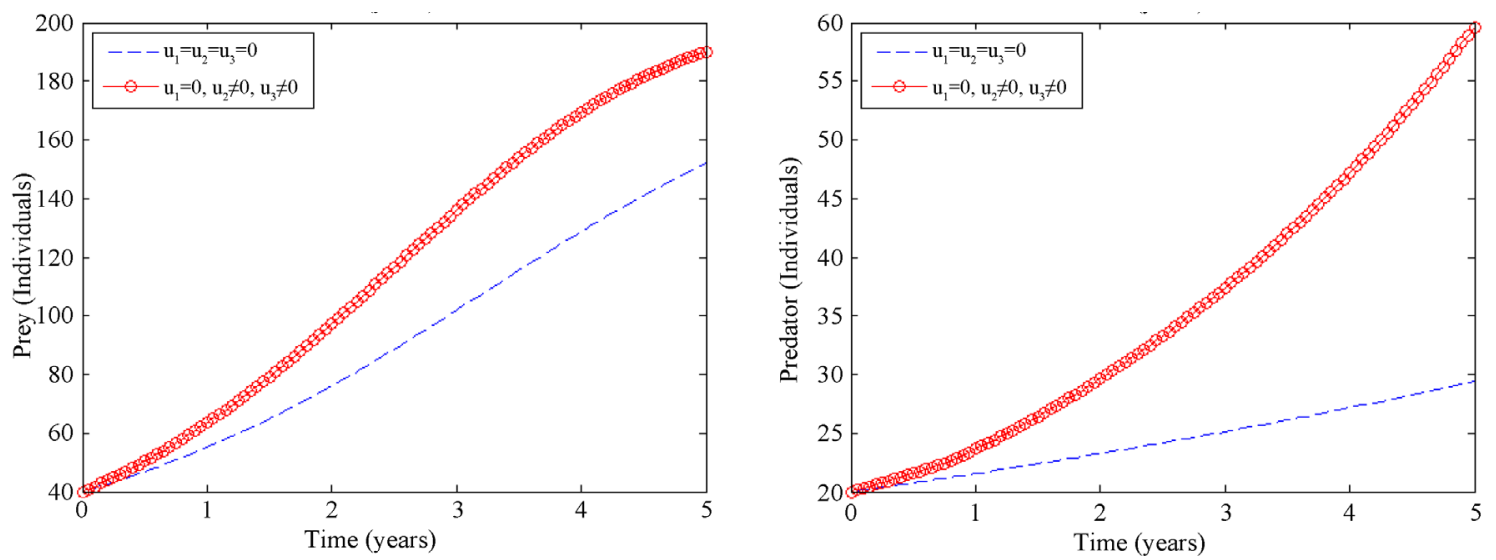

Figure 6. Simulations of a threatened prey-predator model showing the effect of optimal construction of strong bomas and dams.

\subsection{Strategy G: Combination of Application of Anti-Poaching Patrols, Construction of Strong Bomas and Dams}

Here, all three controls $\left(u_{1}, u_{2}\right.$, and $\left.u_{3}\right)$ are used to optimize the objective function $J$. In Figure 7, the results show a significant difference in prey and predator populations before and after control. From Table 1 numerical results indicate that if no control measures are undertaken the lion population would go to approximately 29 individuals from 20 initial individuals during these 5 years. With this control strategy the lion population rises to 65 which is a saving of 36 individuals. Wildebeest population rises from approximately 152 to 223 which is a saving of 71 individuals. This is the strategy which shows the best result for both species among all others as it yields the highest combination of prey and predator individuals saved as a result of control application.

\section{Concluding Remarks}

In this paper, we presented a threatened prey-predator model using a deterministic system of differential equations. The threats are poaching, retaliatory killings and drought. Controls are introduced to the system which are anti-poaching patrols, construction of strong bomas and dams for controlling poaching, retaliatory killings and drought, respectively. In investigating the effect of optimal control, we use one control at a time, a combination of two controls at a time while setting other(s) to zero to compare the effects of the control strategies on the eradication of threats to prey-predator system. Additionally, the case of all controls was also taken into consideration. Our numerical results suggest that the use of all three controls, anti-poaching patrols, construction of strong bomas and dams has highest impact on the control of the system threats. From these findings, we see that 

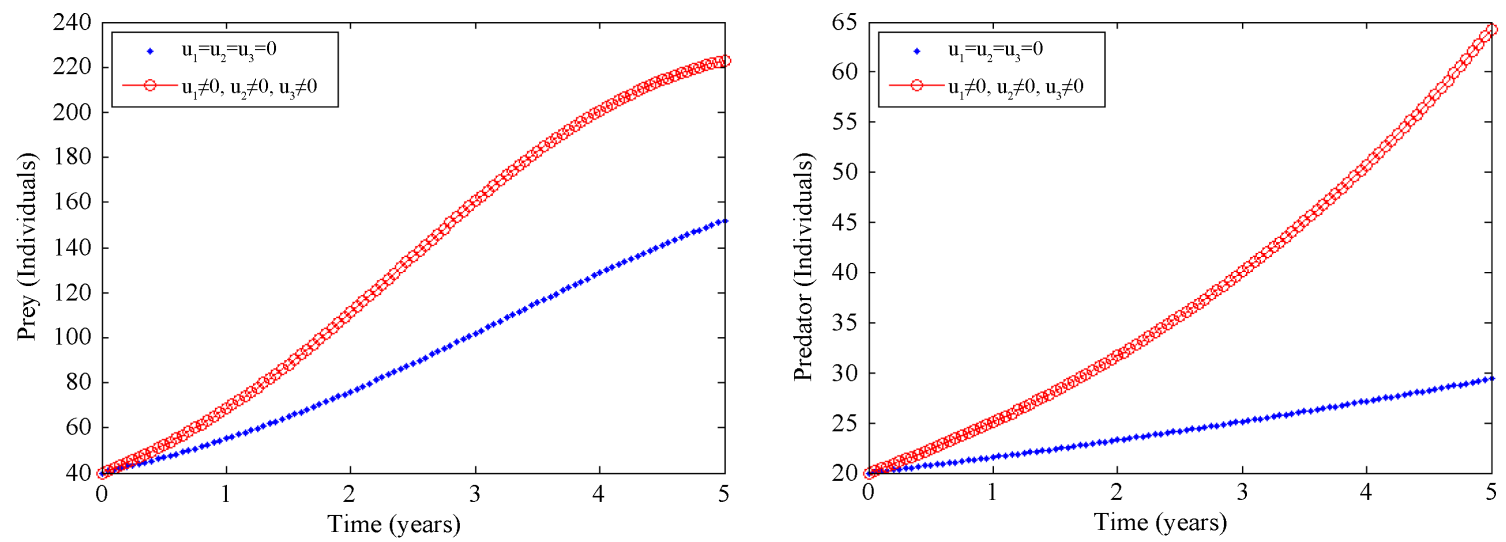

Figure 7. Simulations of a threatened prey-predator model showing the effect of optimal application of anti-poaching patrols, construction of strong bomas and dams.

when we have a system threatened by various threats, dealing with individual threat to the system does not often yield best results. Instead, combined approach of tackling all threats to yield better result is a good approach in the management of the wildlife populations. There is thus a booster effect when tackling many population decimating factors at a go than dealing with them individually. In case of single control strategy implementation, the priority would be construction of dams as it saves more individuals compared to others.

\section{References}

[1] Chakraborty, K., Chakraborty, M. and Kar, T. (2011) Optimal Control of Harvest and Bifurcation of a Prey-Predator Model with Stage Structure. Applied Mathematics and Computation, 217, 8778-8792. http://dx.doi.org/10.1016/j.amc.2011.03.139

[2] Dubey, B. (2007) A Prey-Predator Model with a Reserved Area. Nonlinear Analysis: Modeling and Control, 12, 479494.

[3] Peterson, M.J. (1991) Wildlife Parasitism, Science and Management Policy. Wildlife Management, 55, 782-789. http://dx.doi.org/10.2307/3809532

[4] Walker, B., Carpenter, S., Anderies, J., Abel, N., Cumming, G., Janssen, M., Lebel, L., Norberg, J., Peterson, G. and Pritchard, R. (2002) Resilience Management in Social-Ecological Systems: A Working Hypothesis for a Participatory Approach. Conservation Ecology, 6, 14.

[5] Sinclair, A.R.E., Packer, C., Mduma, S.A.R. and Fryxell, J.M. (2008) Serengeti III, Human Impacts on Ecosystems Dynamics. The University of Chicagopress, Chicago. http://dx.doi.org/10.7208/chicago/9780226760353.001.0001

[6] Kideghesho, J.R. (2010) "Serengeti Shall Not Die": Transforming an Ambition into a Reality. Tropical Conservation Science, 3, 228-248.

[7] Bolger, D., Newmark, W., Morrison, T. and Doak, D. (2008) The Need for Integrative Approaches to Understand and Conserve Migratory Ungulates. Ecology Letters, 11, 63-77.

[8] Haris, G., Thirgood, S., Hopcraft, J., Cromsigt, J. and Berger, J. (2009) Global Decline in Aggregated Migrations of Large Terrestrial Mammals. Endangered Species Research, 7, 55-76. http://dx.doi.org/10.3354/esr00173

[9] GEAS (2013) Saving the Great Migrations: Declining Wildebeest in East Africa? http://na.unep.net/geas/getUNEPPageWithArticleIDScript.php?article id=107

[10] Hopcraft, J., Sinclair, A., Holdo, R., Mwangomo, E., Mduma, S., Thirgood, S., Borner, M., Fryxell, J. and Olff, H. (2013) Why Are Wildebeest the Most Abundant Herbivore in the Serengeti Ecosystem? Serengeti IV: Sustaining Biodiversity in a Coupled Human-Natureal System. University of Chicago Press, Chicago.

[11] Ikanda, D. and Packer, C. (2008) Ritual vs. Retaliatory Killing of African Lions in the Ngorongoro Conservation Area, Tanzania. Endangered Species Research, 6, 67-74. http://dx.doi.org/10.3354/esr00120

[12] Kissui, B.M. (2008) Livestock Predation by Lions, Leopards, Spotted Hyenas and Their Vulnerability to Retaliatory Killing in the Maasai Steppe, Tanzania. Animal Conservation, 11, 422-432.

[13] Kolowski, J.M. and Holekam, K.E. (2006) Spatial, Temporal and Physical Characteristics of Livestock Depradations by Large Carnivores along a Kenyan Reserve Border. Biological Conservation, 128, 529-541. http://dx.doi.org/10.1016/j.biocon.2005.10.021 
[14] Kar, T. and Gosh, B. (2012) Sustainability and Optimal Control of an Exploited Prey-Predator System through Provision of Alternative Food to Predator. Biosystems, 109, 220-232. http://dx.doi.org/10.1016/j.biosystems.2012.02.003

[15] Holling, C.S. (1965) The Functional Response of Predators to Prey Density and Its Role in Mimicry and Popuation Regulation. Memoirs of the Entomological Society of Canada, 97, 1-60. http://dx.doi.org/10.4039/entm9745fv

[16] Fryxell, J.M., Mosser, A., Sinclair, A.R.E. and Packer, C. (2007) Group Formation Stabilizes Predator-Prey Dynamics. Nature, 449, 1041-1043. http://dx.doi.org/10.1038/nature06177

[17] Schaller, G.B. (1972) The Serengeti Lion: A Study of Predator-Prey Relations. University of Chicago Press, Chicago.

[18] Mduma, S.R. (1996) Serengeti Wildebeest Population Dynamics: Regulation, Limitation and Implication for Harvesting. Ph.D. Thesis, University of British Columbia, Canada. 